\title{
Pulmonales kapilläres Blutvolumen und Membrankomponente der pulmonalen Diffusionskapazität bei Patienten mit chronisch obstruktiver Bronchitis (COPD)
}

\author{
Pulmonary Capillary Blood Volume and Membrane Components of Pulmonary Diffusion Capacity in Patients \\ with Chronic Obstructive Bronchitis (COPD)
}

Autoren

Institut
U. Schulz, S. Langwieler, S. Riedel, J. Schreiber

Abteilung für Pneumologie des Universitätsklinikums der Otto-von-Guericke Universität Magdeburg eingereicht 12.1.2014 akzeptiert nach Revision 27.1.2014

Bibliografie

Dol http://dx.doi.org/ 10.1055/s-0034-1365056 Online-Publikation: 10.3.2014 Pneumologie 2014; 68: 266-269 (c) Georg Thieme Verlag KG Stuttgart · New York ISSN 0934-8387

Korrespondenzadresse Prof. Dr. Jens Schreiber Universitätsklinikum Magdeburg Abteilung für Pneumologie Leipziger Straße 44 39120 Magdeburg jens.schreiber@med.ovgu.de

\section{Zusammenfassung}

$\nabla$

Die chronisch obstruktive Bronchitis (COPD) und das Lungenemphysem sind durch eine Einschränkung der Diffusionskapazität charakterisiert. Die relativen Anteile der Teilkomponenten Membranfaktor (Dm) und kapilläres Volumen $(\mathrm{Vc})$ sind bisher unbekannt. Diese Teilkomponenten können mit Hilfe der Messung der NO-Diffusion charakterisiert werden.

Im Rahmen einer prospektiven Studie wurden 183 Patienten mit einer COPD unterschiedlicher Schweregrade lungenfunktionsanalytisch mit der Spirometrie, Bodyplethysmografie und der SingleBreath-Diffusionsmessungen für CO (DLCO) und NO (DLNO) untersucht.

Die DLCO zeigte eine schweregradabhängige Reduktion. Ebenso waren sowohl Dm als auch Vc mit der Einsekundenkapazität $\left(\mathrm{FEV}_{1}\right)$ positiv korreliert. Die Einschränkung von Vc stand im Vergleich zu Dm bei allen Schweregraden der COPD quantitativ im Vordergrund und war bei Patienten mit schwerer COPD statistisch signifikant stärker ausgeprägt.

Es wird geschlussfolgert, dass sowohl Dm als auch Vc schweregradabhängig an der Einschränkung der pulmonalen Diffusionskapazität bei der COPD beteiligt sind mit einem Überwiegen der vaskulären Komponente. Dies stützt pathogenetische Konzepte einer funktionellen Relevanz des Verlustes pulmonaler Kapillaren bei der COPD.

\section{Einleitung}

Die chronisch obstruktive Bronchitis (COPD) ist durch eine chronische, meist progrediente Bronchialobstruktion und Destruktion des Lungenparenchyms mit Ausbildung eines Lungenemphysems charakterisiert [1]. Bei diesem Prozess kommt es neben einem Verlust von Alveolarsepten zu einer Rarifizierung der pulmonalen Kapillaren. Liebow konnte bereits in den fünfziger Jah-

\section{Abstract \\ $\nabla$}

Reduced pulmonary diffusion capacity is a hallmark of COPD, although the relative contribution of the subcomponents of pulmonary diffusion membranous component (Dm) and capillary volume $(\mathrm{Vc})$ - is unknown. These components can be measured with the method of NO single-breath diffusion (DLNO).

In a prospective study, pulmonary function tests including spirometry, body plethysmography and single-breath measurements of diffusion capacity with CO and NO were performed in 183 patients with COPD of varying severity.

There was a severity-dependent decrease in DLCO. Furthermore, Dm as well as Vc was positively correlated with the severity of COPD measured by $\mathrm{FEV}_{1}$. In all stages of COPD, reduction of Vc was more pronounced than constriction of Dm. In patients with most severe COPD, the preponderance of the reduction of Vc was significantly more marked than in milder stages.

We conclude that Dm as well as Vc contributes to the reduction of DLCO in COPD, with a predominance of Vc at all stages of COPD. This confirms the idea that the loss of pulmonary capillaries in COPD is functionally relevant. ren zeigen, dass Lungen, die emphysematös verändert waren, weniger Blutgefäße aufweisen [2]. Weiterhin wurde nachgewiesen, dass der Verlust von Alveolarsepten durch eine gesteigerte Apoptose induziert wird [3] und dass ein Emphysem durch eine relative Verarmung an Blutgefäßen charakterisiert ist [4-6]. Tang et al. entwickelten das Konzept des Verlustes von Kapillaren über den Mechanismus der Apoptose von Endothelzellen beim Emphysem [7]. Kanazawa et al. fanden 
erhöhte Vascular Endothelial Growth Factor (VEGF)-Werte im Sputum von COPD-Patienten und verminderte Werte im Sputum von Emphysempatienten. VEGF war negativ mit dem FEV $_{1}$ bei Patienten mit COPD und positiv bei Patienten mit Emphysem korreliert. Zudem konnten sie eine Assoziation zwischen hohen VEGF-Werten und besserem Gasaustausch, gemessen mit Hilfe der DLCO, zeigen [8]. Diese Befunde sind weitere Hinweise auf eine Relevanz pulmonaler Blutgefäße bei der COPD.

Die strukturellen Veränderungen haben funktionelle Konsequenzen, die mit Hilfe von Lungenfunktionsuntersuchungen quantifizierbar sind. Die etablierten lungenfunktionellen Untersuchungen messen vorwiegend Einschränkungen der Ventilation und global der Diffusion. Veränderungen des kapillären Blutvolumens sind bisher nicht Gegenstand der lungenfunktionsanalytischen Diagnostik. Die Bedeutung dieser Veränderungen ist für Patienten mit COPD nur unzureichend charakterisiert.

Die Diffusionskapazität (DLCO) wird im klinischen Alltag meist mit der Single-Breath-Methode mit Kohlenmonoxid (CO) als Testgas bestimmt. Roughton und Forster [9] erstellten ein Modell, welches beschreibt, dass sich der totale Diffusionswiderstand 1/DLCO aus zwei Widerständen zusammensetzt: dem Widerstand der alveolo-kapillären Membran (1/Dm) und dem reaktiven Widerstand des Blutes in den Alveolarkapillaren $(1 / \theta \times \mathrm{Q} c \times[\mathrm{Hb}])$.

$$
\frac{1}{D L C O}=\frac{1}{D m}+\frac{1}{\theta \times Q c \times[H b]}
$$

Dm = Membranleitfähigkeit, Qc=effektives kapilläres Blutvolumen in $\mathrm{ml},[\mathrm{Hb}]=$ Hämoglobinkonzentration, $\Theta=$ Konstante der CO-Aufnahmerate der Erythrozyten pro $\mathrm{ml}$ normalem Blut in $\mu \mathrm{mol} \times \mathrm{s}^{-1} \times \mathrm{ml}^{-1}[10]$.

Als Methode zur Bestimmung der Membrankomponente (Dm) und des kapillären Blutvolumens (Vc) wurde in den letzten Jahren die Diffusionsmessung mit Stickstoffmonoxid (NO) als Testgas entwickelt (DLNO). Der Untersuchungsablauf ähnelt der DLCO-Messung. DLNO wird während eines Single-Breath-Manövers mit einer identischen Atemanhaltezeit gemessen. Da die Reaktionsrate von Hämoglobin, die nach HB-Korrektur mit dem pulmonalen kapillären Blutvolumen gleichgesetzt wird, mit NO ca. 1400 Mal schneller ist als mit CO, wird DLNO vernachlässigbar von Änderungen des kapillären Blutvolumens beeinflusst. DLNO entspricht somit im Zweikomponentenmodell der pulmonalen Diffusionskapazität dem Membranfaktor Dm und wird nur durch Dm limitiert. Die Differenz zwischen DLCO und DLNO ermöglicht dann eine Berechnung von Vc [11].

Bei einer COPD ist DLCO schweregradabhängig eingeschränkt $[10,14]$. Mit diesen Untersuchungen sollten mit Hilfe der Messung von DLNO zur Berechnung der Membrankomponente Dm und des kapillären Blutvolumens Vc charakterisiert werden, welche Komponenten der Diffusionskapazität an dieser Einschränkung in den einzelnen Stadien beteiligt sind.

\section{Patienten und Methodik}

$\nabla$

Es wurden 183 Patienten mit einer COPD (Diagnostik entsprechend der GOLD-Kriterien [1]) prospektiv in die Untersuchung eingeschlossen. Die Schweregradeinteilung erfolgte $\mathrm{FEV}_{1}$-basiert entsprechend der GOLD-Empfehlungen [1]. Es wurden 27 Patienten mit leichtgradiger (Gruppe 1), 109 Patienten mit mittelgradiger (moderat) (Gruppe 2), 37 mit schwerer (Gruppe 3) und 10 mit sehr schwerer (Gruppe 4) COPD untersucht ( $\bullet$ Tab. 1).

Ausschlusskriterien waren Asthma bronchiale, Herzinsuffizienz ( $\mathrm{EF}<50 \%$ ), akute Lungenarterienembolie, akuter Myokardinfarkt, signifikantes Vitium cordis, andere strukturelle Lungenerkrankungen (Fibrosen u. a.) und die Leberzirrhose.

Es wurden Alter, Geschlecht, Größe, Gewicht, Nikotinkonsum (pack years) sowie Begleiterkrankungen und Medikation dokumentiert. Danach erfolgten lungenfunktionelle Untersuchungen mit Spirometrie, Fluss-Volumen-Kurve und Ganzkörperplethysmografie (MasterScreenPro ${ }^{\circledR}$, Fa. Viasys) entsprechend der üblichen Standards. Die Messungen der Diffusionskapazität erfolgten mit der Single-Breath-Methode für CO und zusätzlich für NO zur Charakterisierung von Dm und Vc (Fa. Viasys MS PFT Pro ${ }^{\circledR}$ ). Die Messwerte wurden in \% der jeweiligen individuellen Sollwerte berechnet. Die in diesem Arbeitsplatz hinterlegten individuellen Sollwerte basieren auf Untersuchungen von Crapo und Crapo [14] und sind auf deren Grundlage teilweise extrapoliert.

Statistik: Es erfolgten eine Varianzanalyse für unabhängige Stichproben, Mehrfachvergleiche nach Bonferroni und eine Korrelationsanalyse nach Pearson nach vorangegangener Testung auf Normalverteilung. Eine Irrtumswahrscheinlichkeit ( $\alpha$-Fehler) von $\mathrm{p}<0.05$ wurde als statistisch signifikant betrachtet. Bei multiplen Korrelationen erfolgte eine entsprechende Adjustierung des Signifikanzniveaus. Die statistische Auswertung erfolgte mit Hilfe des Programms WinSTAT ${ }^{\circledR}$.

\section{Ergebnisse \\ $\nabla$}

Die pulmonale Diffusionskapazität (DLCO) zeigte eine schweregradabhängige Abnahme ( $\bullet$ Abb.1a und $\bullet \mathbf{1 b}$ ). Ebenso nahmen sowohl der Membranfaktor (Dm) als auch das kapilläre Blutvolumen (Vc) schweregradabhängig ab. Beide Teilkomponenten der pulmonalen Diffusionskapazität korrelierten statistisch hoch signifikant positiv mit dem $\mathrm{FEV}_{1}$. Mit abnehmendem $\mathrm{FEV}_{1}$ nehmen auch Dm und Vc ab. So betrug für die Korrelation zwischen $\mathrm{FEV}_{1}$ und Dm r = 0,52 ( $\mathrm{p}<0,001)$ und für die Korrelation zwischen $\mathrm{FEV}_{1}$ und Vc $r=0,47(p<0,001)$. Es bestanden statistisch signifikante Unterschiede sowohl für Dm als auch für Vc zwischen den Gruppen 1 und 2 bzw. 1 und 3, 1 und 4, 2 und 3 sowie 2 und 4 (p jeweils $<0,001$ ). Hingegen waren keine signifikanten Unterschiede zwischen den Patienten mit schwerer (GOLD III) und mit sehr schwerer COPD (GOLD IV) nachweisbar ( $\bullet$ Abb. 2-3).

Die Quotienten aus der jeweiligen Einschränkung von Dm und Vc $(\mathrm{Dm} / \mathrm{Vc})$ zeigten das Verhältnis der Einschränkungen der einzel-

Tab. 1 Patientencharakteristika.

\begin{tabular}{|lcccc|}
\hline COPD-Schweregrad & leicht & moderat & schwer & sehr schwer \\
\hline Alter & $63,7 \pm 10,1$ & $66,5 \pm 10,6$ & $65,1 \pm 9,2$ & $57,2 \pm 5,8$ \\
\hline Geschlecht $(w / m)$ & $11 / 16$ & $21 / 89$ & $8 / 29$ & $2 / 8$ \\
\hline Größe $(\mathrm{cm})$ & $169,6 \pm 9,3$ & $172,4 \pm 8,9$ & $173,0 \pm 7,5$ & $174,6 \pm 8,3$ \\
\hline Gewicht $(\mathrm{kg})$ & $78,2 \pm 22,0$ & $82,0 \pm 17,5$ & $81,7 \pm 24,0$ & $74 \pm 10,1$ \\
\hline BMI $\left(\mathrm{kg} / \mathrm{m}^{2}\right)$ & $27,0 \pm 6,2$ & $27,6 \pm 5,5$ & $27,4 \pm 8,1$ & $24,7 \pm 4,6$ \\
\hline
\end{tabular}



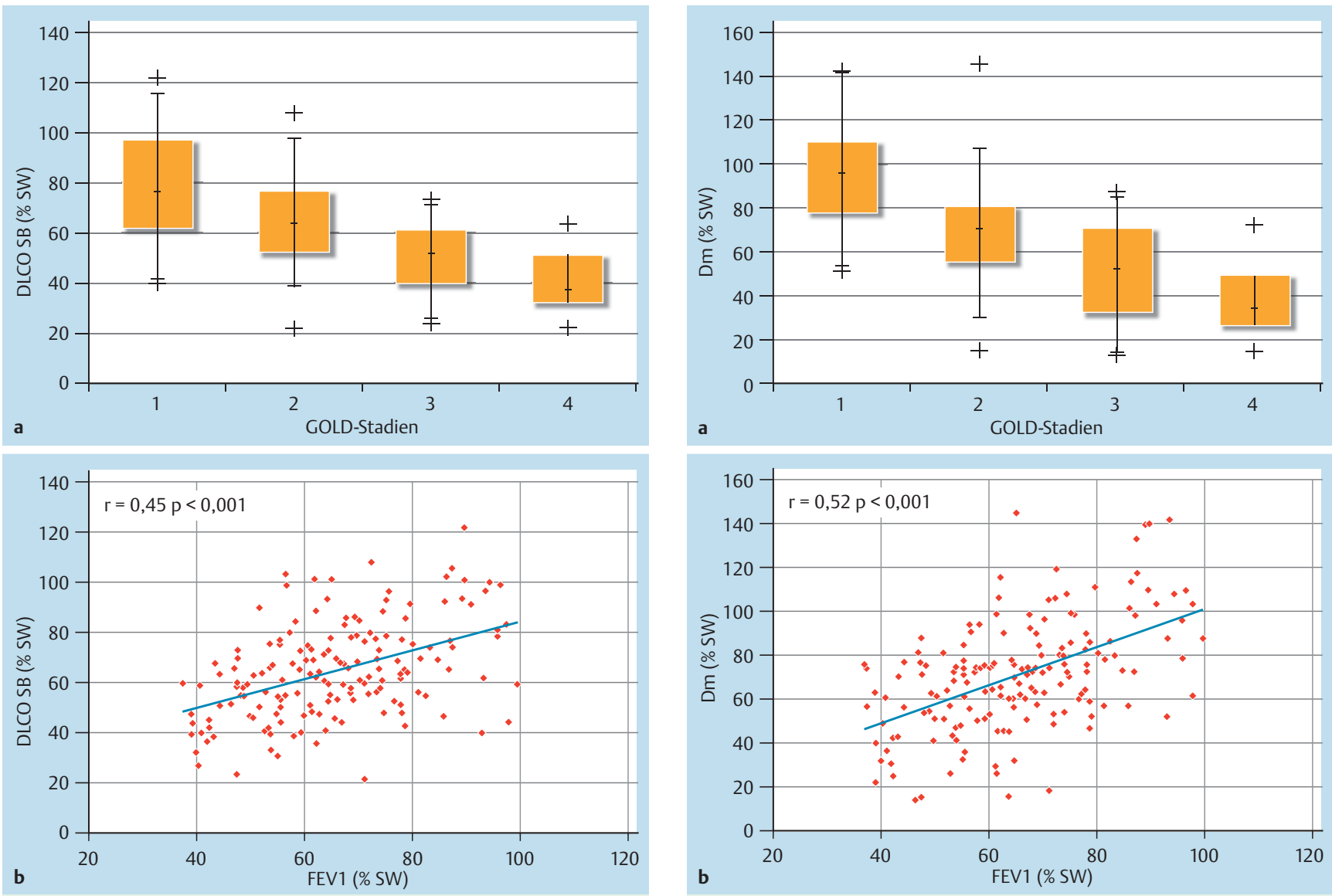

Abb. 1 Pulmonale Diffusionskapazität für CO (DLCO) bei Patienten mit COPD unterschiedlicher Schweregrade (Statistik im Text).

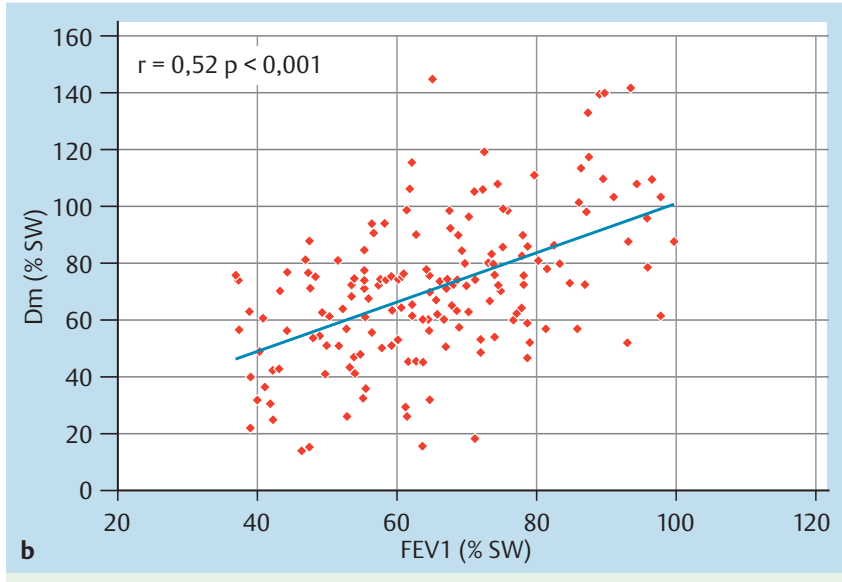

Abb.2 Membrankomponente der pulmonalen Diffusionskapazität (Dm) bei Patienten mit COPD unterschiedlicher Schweregrade (Statistik im Text).

nen Teilkomponenten. Dm/Vc war schwach signifikant negativ mit dem $\mathrm{FEV}_{1}$ korreliert. Mit abnehmendem $\mathrm{FEV}_{1}$ nahm $\mathrm{Dm} / \mathrm{Vc}$ diskret $\mathrm{zu}(\mathrm{r}=-0,12)(\mathrm{p}<0,05)$. Dm/Vc war bei der Mehrzahl der Patienten schweregradunabhängig $>1$. Somit überwiegt schweregradunabhängig die Einschränkung von Vc über die Einschränkung von Dm. Signifikante Unterschiede zugunsten eines Überwiegens der Vc-Einschränkung bestanden zwischen den GOLDGruppen 1 und $4(\mathrm{p}<0,01)$ sowie 2 und $4(\mathrm{p}<0,001)(\bullet$ Abb.4).

\section{Diskussion \\ $\nabla$}

Neben der globalen Diffusionskapazität sind bei der COPD auch die Teilkomponenten der pulmonalen Diffusion, der Membranfaktor (Dm) und das kapilläre Volumen (Vc) schweregradabhängig reduziert. Bereits bei Patienten mit leichtgradiger COPD war eine Einschränkung beider Komponenten der Diffusionskapazität nachweisbar.

Bei der überwiegenden Mehrzahl der Patienten war in allen COPD-Schweregraden das pulmonale kapilläre Volumen (Vc) im Vergleich zum Membranfaktor (Dm) stärker eingeschränkt. Die Reduktion des kapillären Volumens Vc trägt demnach bei Patienten mit COPD unabhängig vom Schweregrad der Erkrankung zur Reduktion der gesamten Diffusionskapazität stärker bei als der Membranfaktor. Das Überwiegen der Vc-Einschränkung gegenüber der Dm-Reduktion war bei den Patienten mit schwerer COPD (GOLD-Stadium 4) im Vergleich zu den leichtergradig erkrankten (GOLD 1 und 2) statistisch signifikant stärker ausge-

prägt. Der von mehreren oben genannten Arbeitsgruppen morphologisch gezeigte und pathogenetisch begründete Aspekt des Verlustes von pulmonalen Kapillaren bei COPD und Emphysem $[2,4-6]$ hat, wie diese Ergebnisse zeigten, für die Patienten eine funktionelle Konsequenz. Die vaskuläre Komponente der Diffusionseinschränkung ist besonders bei Patienten mit schwerer COPD überwiegend.

Die in der vaskulären Theorie zur Entstehung des Emphysems [2] im Fokus stehende Rarifizierung pulmonaler Kapillaren wird hinsichtlich der funktionellen Konsequenz durch die Ergebnisse der Funktionsdiagnostik gestützt. Die u.a. von Santos et al. beschriebene Steigerung der VEGF-Konzentration im Sputum bei Patienten mit leichtgradiger COPD könnte einen reparativen Mechanismus vermuten lassen. Mit unseren Daten kann nicht ausgeschlossen werden, dass die in dieser Arbeit vermutete Vermehrung der Kapillaren in der Bronchialschleimhaut stattfindet [12] und dass sich entgegengesetzt im Lungenparenchym die in ihrer funktionellen Konsequenz beobachtete kapilläre Rarifizierung abspielt. Die relative Kontribution von Gefäßuntergang und -neubildung in Abhängigkeit von den betroffenen Kompartimenten bleibt zukünftigen Untersuchungen vorbehalten.

Eine Limitierung der vorliegenden Untersuchungen ist die geringere Patientenzahl in der Gruppe der schwergradigen COPD. Dies war durch die bekannte Tatsache bedingt, dass es diesen Patienten aufgrund der erforderlichen Atemanhaltezeit und Vitalkapazität oft nicht möglich ist, die Untersuchung korrekt durchzuführen. Wir sehen die prinzipielle Aussage der Untersuchungen dadurch aber nicht eingeschränkt. 

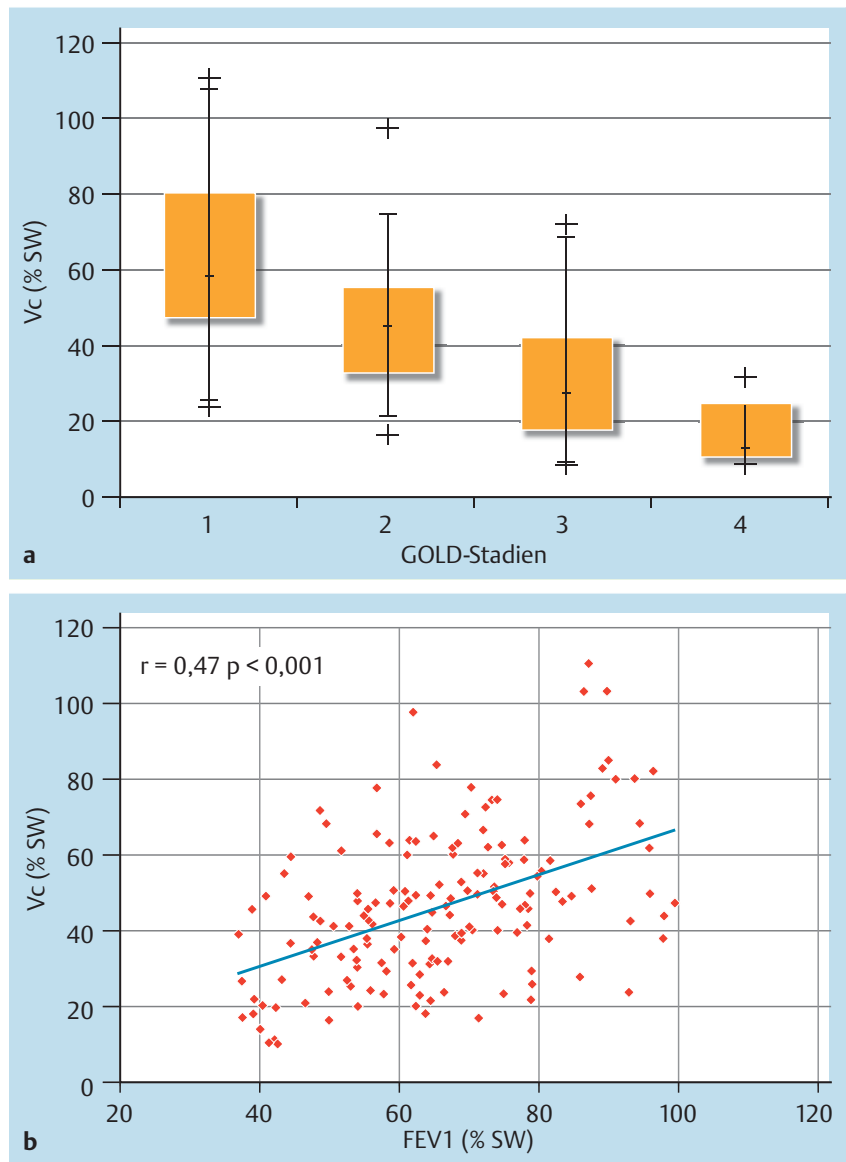

Abb.3 Pulmonales kapilläres Blutvolumen (Vc) bei Patienten mit COPD unterschiedlicher Schweregrade (Statistik im Text).

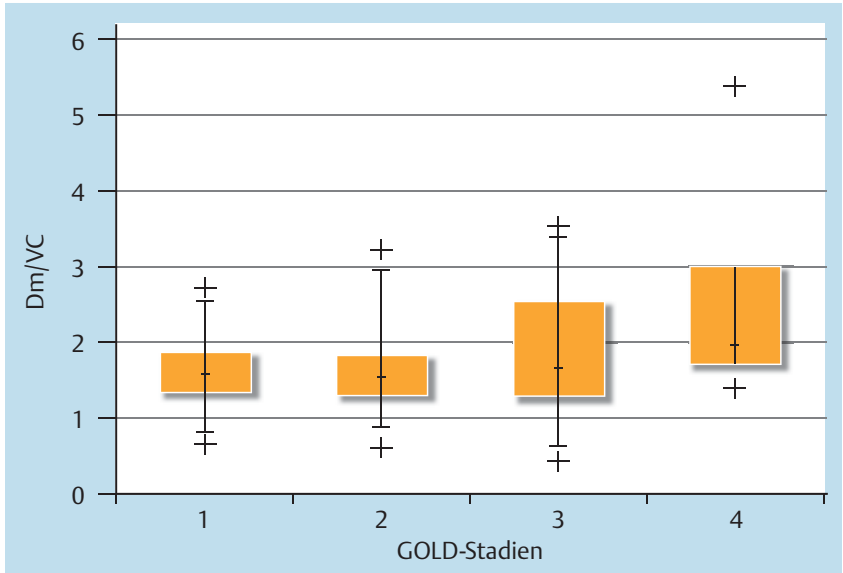

Abb.4 Verhältnis der Einschränkung des pulmonalen kapillären Blutvolumens und der Einschränkung der Membrankomponente der pulmonalen Diffusionskapazität $(\mathrm{Dm} / \mathrm{Vc})$ bei Patienten mit COPD unterschiedlicher Schweregrade (Statistik im Text).

Der Stellenwert der NO-Diffusionsmessung und der Charakterisierung der Teilkomponenten der Diffusion in der klinischen Praxis ist noch unklar $[14,15]$. Erste Ergebnisse sprechen für einen möglichen Einsatz der NO-Diffusionsmessung in der sehr frühen Emphysemdiagnostik bei Rauchern [16]. Diese wird durch unsere Untersuchungen gestützt, dass selbst bei leichtgradiger COPD Veränderungen von Vc und Dm nachweisbar sind. Potenzielle weitere Einsatzgebiete könnten u.a. die Verlaufsbeurteilung von
Patienten mit kardialen und pulmonalen Einschränkungen sein. Dies bleibt jedoch zukünftigen Untersuchungen vorbehalten.

\section{Schlussfolgerung}

Die schweregradabhängige Einschränkung der Diffusionskapazität bei Patienten mit einer COPD ist sowohl durch eine Reduktion der Membrankomponente als auch des kapillären Blutvolumens bedingt. Diese Veränderungen sind bei allen Schweregraden der COPD nachweisbar und korrelieren mit dem FEV ${ }_{1}$. Die Reduktion des pulmonalen kapillären Blutvolumens steht bei allen Schweregraden der COPD quantitativ im Vordergrund.

\section{Interessenkonflikt}

Alle Autoren geben an, dass keine Interessenkonflikte hinsichtlich dieser Untersuchungen bestehen.

\section{Literatur}

1 Decramer M, Vestbo J, Hui DSC et al. Global Initiative for Chronic Obstructive Lung Disease. Pocket Guide to COPD Diagnosis, Management, and Prevention. 2013. http://goldcopd.org/uploads/users/files/ GOLD_2013Feb13.pdf

2 Liebow A. Pulmonary emphysema with special emphasis to vascular changes. Am Rev Respir Dis 1959; 80: 67-93

3 Kasahara Y, Tuder RM, Cool CD et al. Endothelial cell death and decreased expression of vascular endothelial growth factor and vascular endothelial growth factor receptor 2 in emphysema. Am J Respir Crit Care Med 2001; 163: 737-744

4 Voelkel NF, Douglas I, Nicolls M. Angiogenesis in Chronic Lung Disease. Chest 2007; 131: 874-879

5 Wiebe BM, Laursen I. Lung morphometry by unbiased methods in emphysema: bronchial and blood vessel volume, alveolar surface and capillary length. APMIS 1998; 106: 651-656

6 Kasahara Y, Tuder RM, Taraseviciene-Steward $L$ et al. Inhibition of VEGF receptors causes lung cell apoptosis and emphysema. J Clin Invest 2000; 106: 1311 - 1319

7 Tang $K$, Rossiter $H$, Wagner $P$ et al. Lung-targeted VEGF inactivation leads to an emphysema phenotype in mice. J Appl Physiology 2004; 97: $1559-1566$

8 Kanazawa H, Asai K, Hirata Ket al. Possible effects of vascular endothelial growth factor in the pathogenesis of chronic obstructive pulmonary disease. Am J Med 2003; 114: 354 - 358

9 Roughton FJ, Forster R. Relative importance of diffusion and chemical reaction rates in determing the rate of exchange of gases in the human lung, with special reference to true diffusion capacity of pulmonary membrane and volumes of blood in the lung capillaries. J Appl Physiol 1957; 11: 290-302

10 Horstman $M$, Mertens F, Stam $H$. Transfer factor for carbon monoxide. Eur Respir Mon 2005; 31: 127-145

11 Van der Lee I, Zanen P, Stigner $N$ et al. Diffusing capacity for nitric oxide: Reference values and dependence on alveolar volume. Respir Med 2007; 101: 1579-1584

12 Santos S, Peinado VI, Ramirez J. Enhanced expression of vascular endothelial growth factor in pulmonary arteries of smokers and patients with chronic obstructive pulmonary disease. Am J Respir Crit Care Med 2003; 167: 1250-1256

13 Crapo JD, Crapo RO. Comparision of total lung diffusion capacity and the membrane component of diffusion capacity as determined by physiologic and morphometric techniques. Respir Physiol 1983; 51: 183-194

14 Artmann F, Hader C, Rühle KH et al. Die Diffusionskapazität in der täglichen Praxis. Atemw.-Lungenkht 2009; 35: 10-17

15 Van der Lee I, Zanen P, van den Bosch $M$ et al. Pattern of diffusion disturbance related to clinical diagnosis: The $\mathrm{K}(\mathrm{CO})$ has no diagnostic value next to DL(CO). Respir Med 2006; 100: 101-109

16 Van der Lee I, Gieterna HA, Zanen P et al. Nitric oxide diffusing capacity versus spirometry in the early diagnosis of emphysema in Smokers. Respir Med 2009; 103: 1892 - 1897 